\title{
In Vivo Kinematic Analysis of Bicruciate-retaining Total Knee Arthroplasty Focused on Function of the ACL
}

Kosei Ishigaki ( $\nabla$ kousei.ishigaki@med.toho-u.ac.jp )

Toho University - Omori Campus: Toho Daigaku https://orcid.org/0000-0002-6300-6085

Hideyuki Aoki

Toho University Omori Medical Center: Toho Daigaku Iryo Center Omori Byoin

Ryo Takamatsu

Toho University Omori Medical Center: Toho Daigaku Iryo Center Omori Byoin

Yuji Nishiwaki

Toho University: Toho Daigaku

Hiroshi Takahashi

Toho University Omori Medical Center: Toho Daigaku Iryo Center Omori Byoin

Takashi Nakamura

Toho University Omori Medical Center: Toho Daigaku Iryo Center Omori Byoin

Research article

Keywords: Total knee arthroplasty (TKA), kinematic analysis, bicruciate-retaining, ACL, In vivo

Posted Date: February 10th, 2021

DOl: https://doi.org/10.21203/rs.3.rs-180548/v1

License: (9) (i) This work is licensed under a Creative Commons Attribution 4.0 International License.

Read Full License 
In vivo kinematic analysis of bicruciate-retaining total knee arthroplasty focused on function of the ACL

Kosei Ishigaki ${ }^{1}$, Hideyuki Aoki ${ }^{1}$, Ryo Takamatsu ${ }^{1}$, Yuji Nishiwaki ${ }^{2}$, Hiroshi Takahashi ${ }^{1}$, Takashi Nakamura ${ }^{1}$

${ }^{1}$ Department of Orthopedic Surgery, Faculty of Medicine, Toho University

${ }^{2}$ Department of Environmental and Occupational Health, Faculty of Medicine, Toho University

Corresponding author: Kosei Ishigaki

Toho University School of Medicine

6-11-1 Omori-nishi Ota-ku Tokyo, Japan

Zip-code 143-8541

TEL +81-3-3762-4151, FAX +81-3-3763-7539, E-mail: kousei.ishigaki@,med.tohou.ac.jp

Hideyuki Aoki: aokin8aokin8@gmail.com

Ryo Takamatsu: ryou.takamatsu@med.toho-u.ac.jp

Yuji Nishiwaki: yuuji.nishiwaki@med.toho-u.ac.jp

Hiroshi Takahashi: drkan@med.toho-u.ac.jp

Takashi Nakamura: takashin@med.toho-u.ac.jp

Conflict of interest: none declared

This research did not receive any specific grant from funding agencies in the public, commercial, or not-for-profit sectors. 


\section{Introduction}

Total knee arthroplasty (TKA) is an effective surgical procedure for pain and functional disorder caused by severe osteoarthritis of the knee and rheumatoid arthritis, but patient satisfaction remains at about 80\% [1-4]. Conventional TKA does not reproduce normal knee joint movement because the anterior cruciate ligament (ACL) alone or both the ACL and posterior cruciate ligament (PCL) are resected, which reduces patient satisfaction. Bicruciate retaining total arthroplasty (BCR-TKA) preserving both the PCL and ACL was developed because it reproduces a more physiological knee joint movement compared with that reproduced by current TKA preserving only the PCL (CR-TKA).

BCR-TKA is expected to reproduce physiological kinematics close to those of normal knees and to improve stability, compared with CR-TKA. It has been shown that in vivo kinematics after BCR-TKA are similar to those of the normal knee [5].

However, there is no consistent view of the function or advantage of ACL retention by BCR-TKA, and various measures have been used to evaluate postoperative kinematics. In this study, kinematics after BCR-TKA and CR-TKA were analyzed using 2D-3D registration, with the hypothesis that $\mathrm{BCR}-\mathrm{TKA}$ retaining the ACL is superior to $\mathrm{CR}$ TKA for braking performance in the anteroposterior direction.

Regarding the function of the ACL, a comparison of reconstructed knees and the healthy side showed only weak stress on the ACL while walking on level ground, whereas measurements while walking on a downward slope were useful to detect differences in kinematics [6]. Walking on a downward slope has also been used to compare ACL-retaining unicompartmental knee arthroplasty and CR-TKA [7]. Therefore, in this study, deep flexion on level ground and on a $10^{\circ}$ forward slope with a shearing force on the ACL were compared. This is the first in vivo kinematic analysis of BCR-TKA on a forward slope.

\section{Subjects and Methods}

\subsection{Subjects}

The subjects were patients treated with TKA at our hospital between April 2016and March 2018, 10 patients (10 knees) (BCR: 5 patients ( 5 knees), CR: 5 patients ( 5 knees)) with a 6-month or longer course after surgery who could crouch from a standing position to deep flexion. In the BCR group, a Vanguard $\mathrm{XP}^{\circledR}$ (Zimmer Biomet, Warsaw, IN) was used in 3 males ( 3 knees) and 2 females ( 2 knees), and in the CR group, a Triathlon $^{\circledR}$ (Stryker, Mahwah, NJ) was used in 2 males ( 2 knees) and 3 females ( 3 
knees). All cases were treated for osteoarthritis of the knee of KL classification 3 or 4 and the ACL was confirmed to be intact on preoperative MRI. The anterior drawer test and Lachman test were negative in all cases. The preoperative hip-knee-ankle (HKA) angle was $184 \pm 2.3^{\circ}$ in the BCR group and $184.4 \pm 3.4^{\circ}$ in the CR group, and the postoperative HKA angle was $176.8 \pm 1.8^{\circ}$ in the BCR group and $176 \pm 2.5^{\circ}$ in the $\mathrm{CR}$ group. Cases with severe genu varum with a preoperative HKA angle exceeding $190^{\circ}$ were excluded. The Knee Society Score (KSS) was used as the clinical score. The preoperative KSS was $47.4 \pm 4.6$ in the BCR group and $45.8 \pm 3.1$ in the CR group, and the postoperative KSS was $85 \pm 2.7$ in the BCR group and $83.8 \pm 1.6$ in the CR group. Surgery was performed with a medial parapatellar approach by the same surgeon in all cases. The ACL was macroscopically confirmed to be intact during surgery in all patients. Ages at the time of examination (mean \pm standard deviation) were $74.2 \pm 2.2$ and 72.8 \pm 4.3 years and the postoperative follow-up periods were 17.6 \pm 5.1 and 14.6 \pm 5.8 months in the BCR and CR groups, respectively (Table 1). Subjects practiced flexion motion several times, and then the maximum flexion on level ground and a $10^{\circ}$ forward slope was imaged by fluoroscopy to acquire lateral views (Fig. 1). An explanation of the objectives of the study was given to the subjects prior to obtaining written consent, and the study was initiated after approval by the Toho University Faculty of Medicine Ethics Committee (approval number A17041).

\subsection{Imaging}

Images of the knee joint during motion were acquired from the lateral direction at a speed of 12.5 frames/sec using a fluoroscopic apparatus (Curevista ${ }^{\circledR}$; Hitachi Medical Systems Ltd., Japan). The size of the acquisition and collection field of view was $17 \times 17$ inch , and the image size was $1,024 \times 1,024$ pixels. To prepare a $3 \mathrm{D}$ bone model for image matching, the full lengths of the bilateral lower limbs were imaged by CT before surgery, setting the slice interval at $1 \mathrm{~mm}$. From the acquired CT data, 3D femoral and tibial models were prepared using 3D visualization and measurement software, Zed View ${ }^{\mathrm{TM}}$ (LEXI, Tokyo, Japan).

\subsection{Analysis}

Fluoroscopic images were analyzed with an image analysis program, Knee Motion $^{\mathrm{TM}}$ (LEXI, Tokyo, Japan), using 2D-3D image matching. X-rays images were acquired from the hip to ankle joint in the standing position after surgery from two directions: the front and a $60^{\circ}$ oblique angle, and 3D coordinates were prepared from these images. Three 3D images were prepared by matching projected images of the 
bone model with the 3D shape prepared from preoperative CT images with the X-ray images in the two directions [8]. Then, the 3D models of the femoral and tibial components were read and similarly matched. The 3D model prepared using Knee Motion $^{\mathrm{TM}}$ was matched with the fluoroscopic images and the positional relationship between the components was determined (Fig. 2). The estimated accuracy of relative motion between the metal components was $\leq 0.4^{\circ}$ for rotation and $\leq 0.8 \mathrm{~mm}$ for translation [9].

\subsection{Evaluation method}

Rotation kinematics were evaluated based on the rotation angle of the femoral component relative to the tibial component. The angle was defined relative to the angle between the femoral and tibial axes, which was taken to be $0^{\circ}$ (flexion angle $0^{\circ}$ ) to correct for variation due to the implant installation angle. Knee rotations were described using the joint rotational convention of Grood and Suntay [10]. External rotation of the femoral component was defined as positive and internal rotation as negative. Anteroposterior translation and kinetics were evaluated at the point where the medial and lateral contact points of the geometric center axis (GCA) were projected (the most distal point of the femoral component). Posterior translation from the tibial axis was defined as positive and anterior translation as negative (Fig4). The GCA is a line that approximately connects the spherical medial and lateral posterior condyles of the femur (Fig.3).

Statistical analysis was conducted by Mann-Whitney U test to compare the rotational angle, the location of lateral and medial contact points per flexion angle between the BCR group and the CR group. Differences in these parameters between the $\mathrm{BCR}$ and $\mathrm{CR}$ groups across the flexion angles were compared by repeated measures ANOVA. $\mathrm{P}<0.05$ was considered to be statistically significant.

\section{Results}

The mean flexion angles were $116 \pm 13.1^{\circ}$ and $125 \pm 2.7^{\circ}$ and the mean extension angles were $5.4 \pm 4.3^{\circ}$ and $2.0 \pm 3.0^{\circ}$ in the BCR and CR groups, respectively. The femoral component showed gradually external rotation relative to the tibial component with flexion on level ground and on a $10^{\circ}$ forward slope. On level ground, the external rotation at maximum flexion were $14.5 \pm 1.9^{\circ}$ in the BCR group and $7.9 \pm 0.4^{\circ}$ in $\mathrm{CR}$ group (Fig. 5a). By the repeated measures ANOVA, the rotation across the flexion angles was larger in the BCR group with a statistical significance $(\mathrm{p}=0.04)$. In flexion from $0^{\circ}$ to $120^{\circ}$, the medial contact point at the most distal end of the femoral component translated posteriorly by $4.1 \pm 1.7 \mathrm{~mm}$ in the BCR group and by $2.5 \pm 1.6 \mathrm{~mm}$ 
in the CR group. Over all flexion positions, the medial contact point was positioned more anterior in the BCR group with a statistical significance $(p=0.02)$ (Fig. 6a). In contrast, in flexion from $0^{\circ}$ to $120^{\circ}$, the lateral contact point translated posteriorly by $13.2 \pm 1.6 \mathrm{~mm}$ in the BCR group and $7.1 \pm 1.7 \mathrm{~mm}$ in the CR group. Over all flexion positions, the lateral contact point was positioned anterior in the BCR group, but without a statistical significance $(\mathrm{p}=0.23)$ (Fig. $7 \mathrm{a})$.

On a forward slope, in flexion from $0^{\circ}$ to $120^{\circ}$, external rotation was $12.7 \pm 1.5^{\circ}$ in the $\mathrm{BCR}$ group and $7.6 \pm 0.7^{\circ}$ in the $\mathrm{CR}$ group, and over all flexion positions the rotation angle was larger in the BCR group, but it did not reach a statistical significance $(p=0.15)$ (Fig. 5b). In flexion from $0^{\circ}$ to $120^{\circ}$, the medial contact point at the most distal end of the femoral component translated posteriorly by $5.9 \pm 1.8 \mathrm{~mm}$ in the BCR group and $2.9 \pm 0.8 \mathrm{~mm}$ in the $\mathrm{CR}$ group. Over all flexion positions, the installed medial contact point was positioned statistically significantly more anterior in the BCR group $(p=0.02)$ (Fig. 6b). In flexion from $0^{\circ}$ to $120^{\circ}$, the lateral contact point translated posteriorly by $13.6 \pm 1.8 \mathrm{~mm}$ in the BCR group and $7.4 \pm 0.9 \mathrm{~mm}$ in the CR group, and over all flexion positions, the installed lateral point was positioned more anterior in the BCR group, but without a statistical significance $(\mathrm{p}=0.12)$ (Fig. $7 \mathrm{~b})$.

In detailed kinetic data by flexion position, the BCR group on level ground had $14.3^{\circ}$ of lateral rotation at $0^{\circ}$ to $90^{\circ}$ flexion, whereas the CR group had internal rotation at $0^{\circ}$ to $20^{\circ}$ flexion and then $8^{\circ}$ of lateral rotation until $110^{\circ}$ (Fig. 5a). The medial contact point was posterior $2 \mathrm{~mm}$ at $0^{\circ}$ to $90^{\circ}$ flexion in the BCR group, but posterior $8.6 \mathrm{~mm}$ in the CR group over this flexion and translated further posterior as flexion angle increased (Fig. 6a). The lateral contact point translated posteriorly $12.5 \mathrm{~mm}$ at $0^{\circ}$ to $120^{\circ}$ flexion in the BCR group, but translated anteriorly $1.4 \mathrm{~mm}$ and posteriorly 8.5 $\mathrm{mm}$ in the CR group (Fig. 7a). The kinematic pathway showed a medial pivot pattern in which the lateral contact point translated posteriorly while the medial contact point stayed until $90^{\circ}$ flexion in the BCR group. In contrast, the femoral component rotated slightly internally until $20^{\circ}$ flexion and then rotated externally, as a result the lateral contact point translated posteriorly in the CR group. On a forward slope, the BCR group had $11^{\circ}$ of external rotation at $0^{\circ}$ to $90^{\circ}$ flexion, whereas the CR group had internal rotation at $0^{\circ}$ to $20^{\circ}$ flexion (similarly to ground level) and then $9^{\circ}$ of external rotation until $100^{\circ}$ flexion (Fig. $5 \mathrm{~b}$ ). The medial contact point was located posteriorly $2 \mathrm{~mm}$ from the baseline at $0^{\circ}$ flexion, translated anteriorly $1 \mathrm{~mm}$ at a low flexion position, and translated $3 \mathrm{~mm}$ posteriorly until $90^{\circ}$ flexion in the BCR group. The medial contact point was located posteriorly $8 \mathrm{~mm}$ from the baseline at $0^{\circ}$ flexion, translated anteriorly $0.5 \mathrm{~mm}$ at a low flexion position, and translated $9 \mathrm{~mm}$ posteriorly until $90^{\circ}$ flexion in 
the CR group (Fig. 6b). The lateral contact point translated posteriorly $13.5 \mathrm{~mm}$ at $0^{\circ}$ to $120^{\circ}$ flexion in the BCR group, but translated anteriorly $1 \mathrm{~mm}$ on average and posteriorly $7.5 \mathrm{~mm}$ until $120^{\circ}$ flexion in the CR group (Fig. $7 \mathrm{~b}$ ).

\section{Discussion}

Screw home movement (SHM) causing steep external rotation of the femoral component has been shown in squat motion of normal knees in extension to mild flexion [11-15], but this has generally been considered to be due to the ACL and joint surface shape. The lateral contact point at the extension position is moved backward in knees with ACL failure, which loses SHM [16] compared with that of normal knees and suggests that the ACL plays a major role in SHM.

In an analysis of TKA using unfixed cadavers, Hamada et al. found that kinematics were close to those of normal knees in flexion motion without loading after replacing the femoral component, but predominantly showed an internal position from the extension position to early flexion when the tibial component was replaced, which reduced the rotation [17]. Arauz et al. [18] performed in vivo kinematic analysis with loading in 29 knees treated with BCR-TKA, in which tibial rotation from extension to mild flexion was small and SHM was not reproduced. The medial pivot shift representing tibial internal rotation centered on the medial side was only $59 \%$, which suggested that ACL preservation alone cannot reproduce kinematics of normal knees and that reproduction of the joint surface shape is important.

In this study, deep flexion on a forward slope that stressed the ACL was analyzed, in addition to in vivo kinetic analysis of BCR-TKA and CR-TKA on ground level. Both groups showed a medial pivot pattern in which the lateral component point translated posteriorly with centering of the medial component, but the rotation angle differed significantly between the groups. In $0^{\circ}$ to $20^{\circ}$ flexion, the component rotated internally in the CR group, but externally in the BCR group. This may be because the femoral component at extension ( $0^{\circ}$ flexion) was located more internally in the BCR group than in the CR group. Therefore, these results provided evidence that conserved ACL function induced motion close to SHM of the normal knee. These results differ from those of previous studies because kinetics near SHM was observed in a nonphysiological articular surface. Nevertheless, the rotation degree on ground level in the BCR group was significantly larger than that in the CR group, but smaller than that of normal rotation [20] and similar to that found by Kawano et al. [24]. Therefore, the nonphysiological form of the articular surface may have had an effect.

Both medial and lateral contact points between components were located anterior at 
$0^{\circ}$ flexion in the BCR group. Conventional CR-TKA produces a contact point at extension that is more posterior than the normal knee due to PCL tension. In addition, there may be imbalance at the intermediate flexion position and paradoxical motion in which the femoral component slides forward against the tibia due to flexion [17]. The femur translates posterior as a normal knee bends. CR-TKA leads to lower anteroposterior braking and induces paradoxical forward motion; consequently, the polyethylene insert wears and the moment arm of the quadriceps femoris muscle is low $[18,19]$. Slight anterior translation was found in the CR group on a forward slope, which was a paradoxical motion leading to decreased posterior translation. Although similar anterior translation was also observed in the BCR group, there was no decreased posterior translation. This was because the preserved ACL function made a contact point at extension near to that of the normal knee and no paradoxical motion occurred, leading to kinetics that were close to normal. Furthermore, the medial contact point was located anterior at extension in the BCR group; consequently, the component translated posterior in parallel with the lateral contact point, showing bicondylar rollback.

Kinematics close to those of normal knees after BCR-TKA compared with those after CR-TKA have been reported [22-24], but with no significant difference in clinical outcome [27-29]. One study showed that the advantage of ACL preservation was balance perception consistent with a normal knee [26], and these results show the value of BCR-TKA. We note that we conducted in vivo kinetic analysis using Vanguard $\mathrm{XP}{ }^{\circledR}$ in this study. Journey ${ }^{\mathrm{TM}}$ II XR (Smith \& Nephew, Memphis, TN), a newer device, reproduces the physiological form of the articular surface. Therefore, it is expected to provide kinetics close to those of the normal knee and greater satisfaction for patients.

This study had several limitations. First, it included the small number of subjects and low statistical power because limited cases with BCR-TKA were indicated for surgery, and it would take a large amount of time to analyze the data. Also, we tried to consider adjusting for multiple testing but it was not performed because of the small sample size. Second, we investigated only the postoperative kinetic analysis not preoperative kinetics. Third, surgical procedures have an effect on kinematics and different implant design makes it difficult to evaluate the function of the ACL alone. Fourth, there are no criteria for assessing the degree of ACL degeneration and further studies are needed in more subjects, including those before BCR-TKA. Finally, further discussion is required in the future to elucidate if a statistically significant status can be interpreted as a clinically significant status. In addition, the follow-up period ranged from 6 months to one year, but kinetics may change over longer periods. 


\section{Conclusion}

We conducted in vivo kinetic analysis of deep flexion motion on level ground and on a forward slope to stress the ACL. In the BCR group, rotation on level ground was larger and the medial contact point was anterior compared to those in the CR group. These findings suggest kinematics that were close to those of the normal knee in the BCR group.

\section{References}

1. Bourne RB, Chesworth BM, Davis AM, Mahomed NN, Charron KD. Patient satisfaction after total knee arthroplasty: Who is satisfied and who is not? Clin Orthop Relat Res. 2010;468 (1) :57-63.

2. Scott CE, Howie CR, MacDonald D, Biant LC. Predicting dissatisfaction following total knee replacement: A prospective study of 1217 patients. J Bone Joint Surg Br. 2010;92 (9) :1253-1258.

3. Shan L. Intermediate and long-term quality of life after total knee replacement: A systematic review and meta-analysis. Journal of bone and joint surgery.American volume. $2015 ; 97$ (2) :156-168.

4. Choi Y. Patient satisfaction after total knee arthroplasty. Knee surgery \& related research. 2016;28 (1) :1-15.

5. Moro-oka T, Moro-oka T, Muenchinger M, Canciani JP, Banks SA. Comparing in vivo kinematics of anterior cruciate-retaining and posterior cruciate-retaining total knee arthroplasty. Knee surgery, sports traumatology, arthroscopy : official journal of the ESSKA. 2007;15 (1) :93-99.

6. R. O’Hara-Plotnik, E. Thorhauer, A. Sivaprakasam, J. Irrgang, F. Fu, S. Tashman. Gait is a poor task choice for identifying kinematic deficits after ACL reconstruction. ORS 2015 annual meeting. 2015 (40):830.

7. Wiik AV. Downhill walking gait pattern discriminates between types of knee arthroplasty: Improved physiological knee functionality in UKA versus TKA. Knee surgery, sports traumatology, arthroscopy: official journal of the ESSKA. 2015;23 (6) :1748-1755.

8. Yamazaki Y, Kobayashi K, Sakaoto M. Accuracy verification of a newly developed turntable X-ray system in three-dimensional leg alignment assessment system. Annual Meeting of Japanese Society for Clinical Biomechanics and Related Research 26, 181186, 2005-10-01. 2005:181-186.

9. Kobayashi K, Tanaka N, Odagawa K, Sakamoto M, Tanabe Y. Image-based matching for natural knee kinematics measurement using single-plane fluoroscopy. . $J$ Jpn Soc Exp Mech. 2009 ( 9) :162-166. 
10. Grood ES. A joint coordinate system for the clinical description of threedimensional motions: Application to the knee. J Biomech Eng. 1983;105(2):136-144. 11. Goodfellow J, O'Connor J. The mechanics of the knee and prosthesis design. J Bone Joint Surg Br. 1978;60-B ( 3) :358-369.

12. Bytyqi D, Shabani B, Lustig S, Cheze L, Karahoda Gjurgjeala N, Neyret P. Gait knee kinematic alterations in medial osteoarthritis: Three dimensional assessment. Int Orthop. 2014;38 ( 6):1191-1198.

13. Ishii Y, Terajima K, Terashima S, Koga Y. Three-dimensional kinematics of the human knee with intracortical pin fixation. Clin Orthop Relat Res. 1997; (343) (343) :144-150.

14. Wretenberg P, Ramsey DK, Nemeth G. Tibiofemoral contact points relative to flexion angle measured with MRI. Clin Biomech （Bristol, Avon) . 2002;17 (6) :477485 .

15. Asano T, Akagi M, Tanaka K, Tamura J, Nakamura T. In vivo three-dimensional knee kinematics using a biplanar image-matching technique. Clin Orthop Relat Res. 2001; (388) :157-66. doi (388) :157-166.

16. Murayama T. Three-dimensional in vivo dynamic motion analysis of anterior cruciate ligament-deficient knees during squatting using geometric center axis of the femur. Journal of orthopaedic science : official journal of the Japanese Orthopaedic Association. 2016;21 (2) :159-165.

17. Hamada D. Native rotational knee kinematics are lost in bicruciate-retaining total knee arthroplasty when the tibial component is replaced. Knee surgery, sports traumatology, arthroscopy : official journal of the ESSKA. 2018;26 (11) :3249-3256. 18. Arauz P, Klemt C, Li mmahakhun S, An S, Kwon YM. Stair climbing and high knee flexion activities in bi-cruciate retaining total knee arthroplasty: In vivo kinematics and articular contact analysis. J Arthroplasty. 2019;34 (3) :570-576.

19. Dennis DA, Komistek RD, Colwell CE,Jr, et al. In vivo anteroposterior femorotibial translation of total knee arthroplasty: A multicenter analysis. Clin Orthop Relat Res. 1998; (356) :47-57. doi (356) :47-57.

20. Dennis DA, Komistek RD, Mahfouz MR. In vivo fluoroscopic analysis of fixedbearing total knee replacements. Clin Orthop Relat Res. 2003; (410) :114-30. doi (410) :114-130.

21. Mahoney OM, McClung CD, dela Rosa MA, Schmalzried TP. The effect of total knee arthroplasty design on extensor mechanism function. J Arthroplasty. 2002;17 (4) :416-421. 
22. Kono K. In vivo three-dimensional kinematics of normal knees during different high-flexion activities. The bone \& joint journal. 2018;100-B (1) :50-55.

23.Lo J. Translational and rotational knee joint stability in anterior and posterior cruciate-retaining knee arthroplasty. The knee. 2011;18 (6) :491-495.

24. Halewood C. Anteroposterior laxity after bicruciate-retaining total knee arthroplasty is closer to the native knee than ACL-resecting TKA: A biomechanical cadaver study. J Arthroplasty. 2015;30 (12) :2315-2319.

25. Kono K. Bicruciate-retaining total knee arthroplasty reproduces in vivo kinematics of normal knees to a lower extent than unicompartmental knee arthroplasty. Knee surgery, sports traumatology, arthroscopy: official journal of the ESSKA. 2019. 26. Jerosch J. [Proprioceptive abilities of surgically and conservatively treated knee joints with injuries of the cruciate ligament]. Unfallchirurg. 1998;101 (1):26-31. 27. Migaud H. [Influence of the tibial slope on tibial translation and mobility of nonconstrained total knee prosthesis]. Rev Chir Orthop Reparatrice Appar Mot. 1996;82 (1) :7-13.

28. Jenny JY. Preservation of anterior cruciate ligament in total knee arthroplasty. Arch Orthop Trauma Surg. 1998;118 (3) : 145-148.

29. Osmani FA. The utility of bicruciate-retaining total knee arthroplasty. Arthroplasty today. 2017;3 (1):61-66. 
Figures
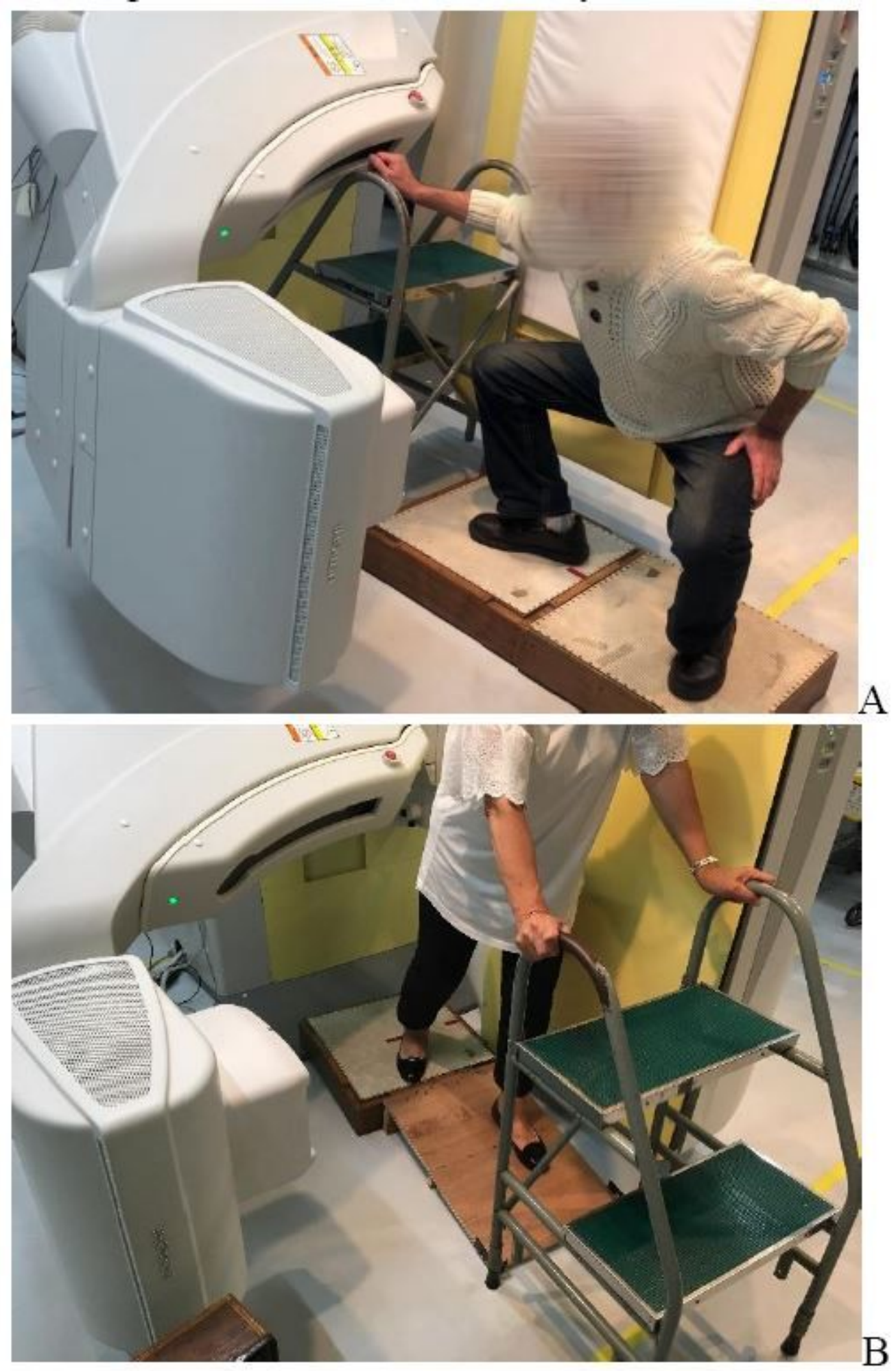

Figure 1

Acquisition of fluoroscopic images of deep flexion (single deep lunges). A: On level ground. B: On a $10^{\circ}$ forward slope. The subjects performed deep flexion while holding on to a handrail for safety. 


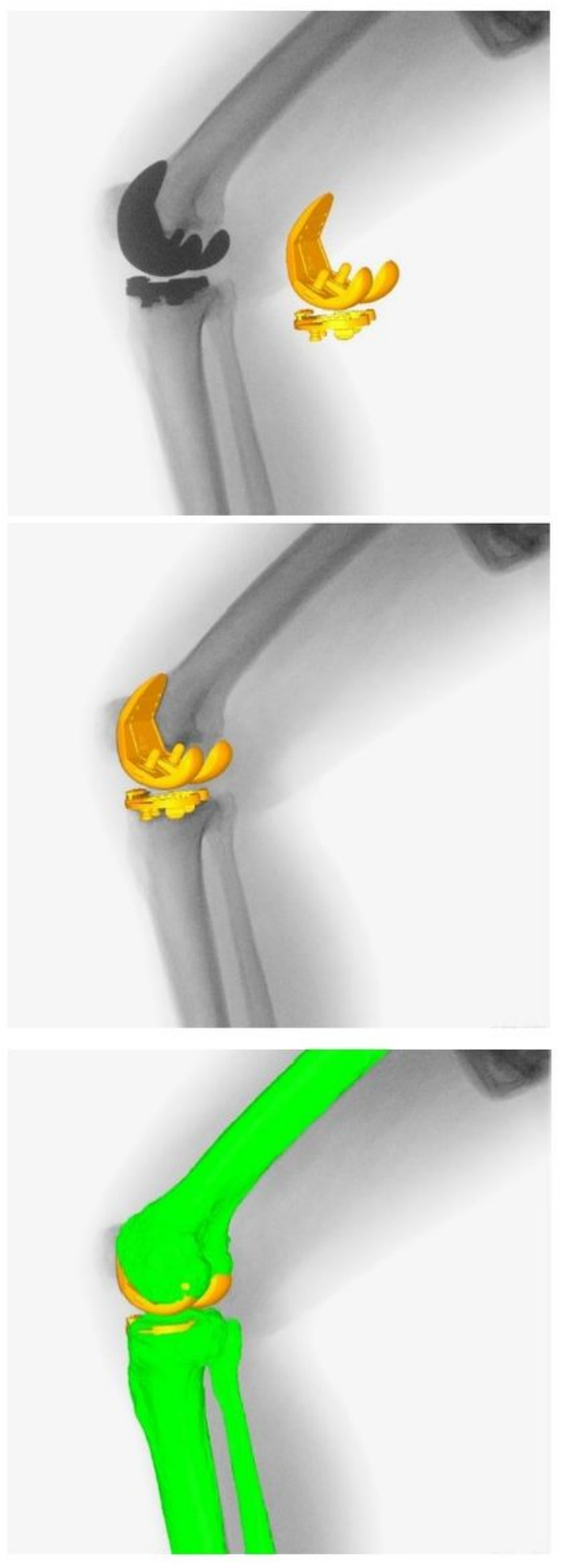

Figure 2

$2 D / 3 D$ registration during a single deep lunge 


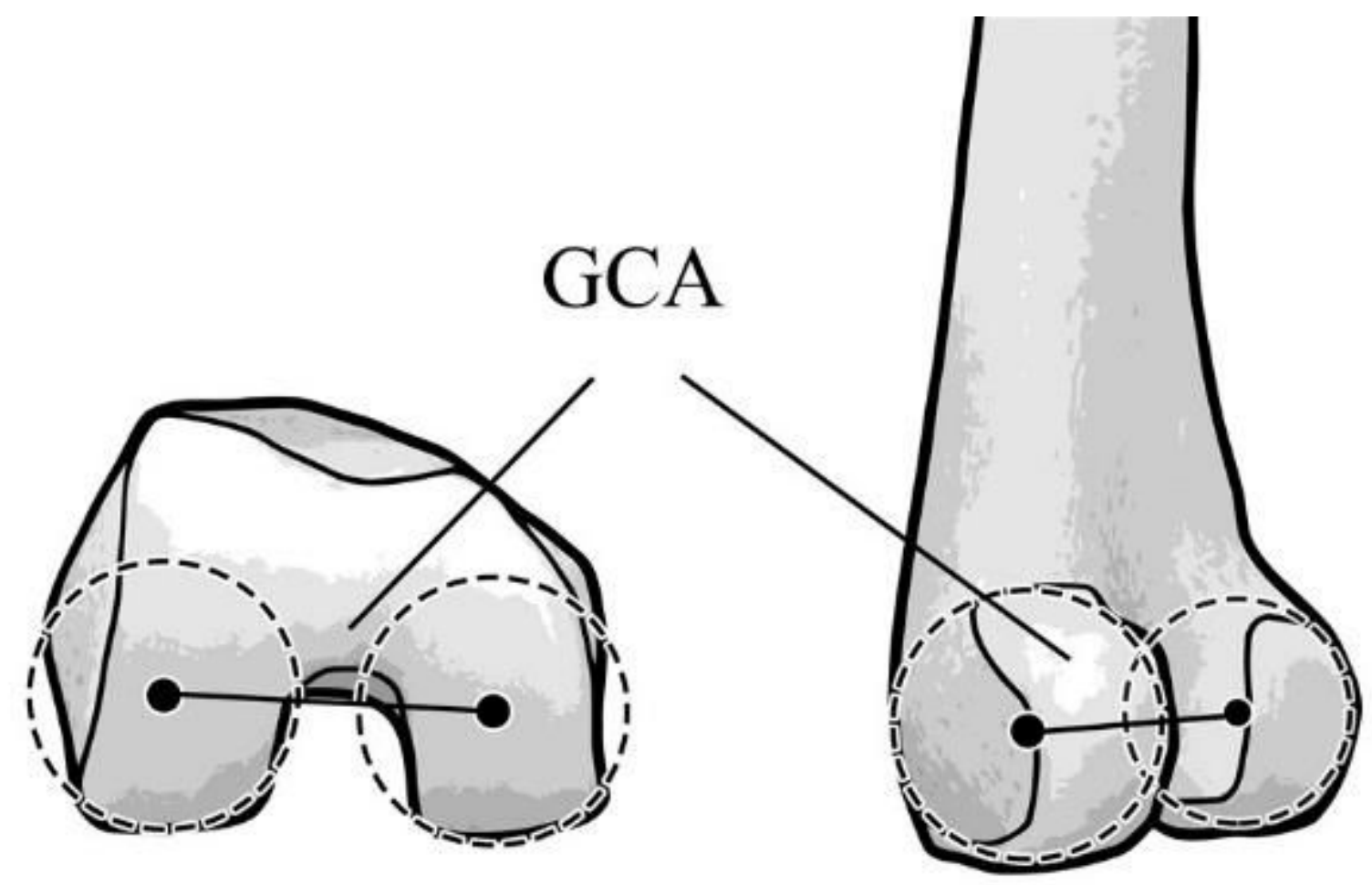

Figure 3

Definition of the geometric center axis (GCA), as a line connecting the approximately spherical medial and lateral posterior condyles of the femur.

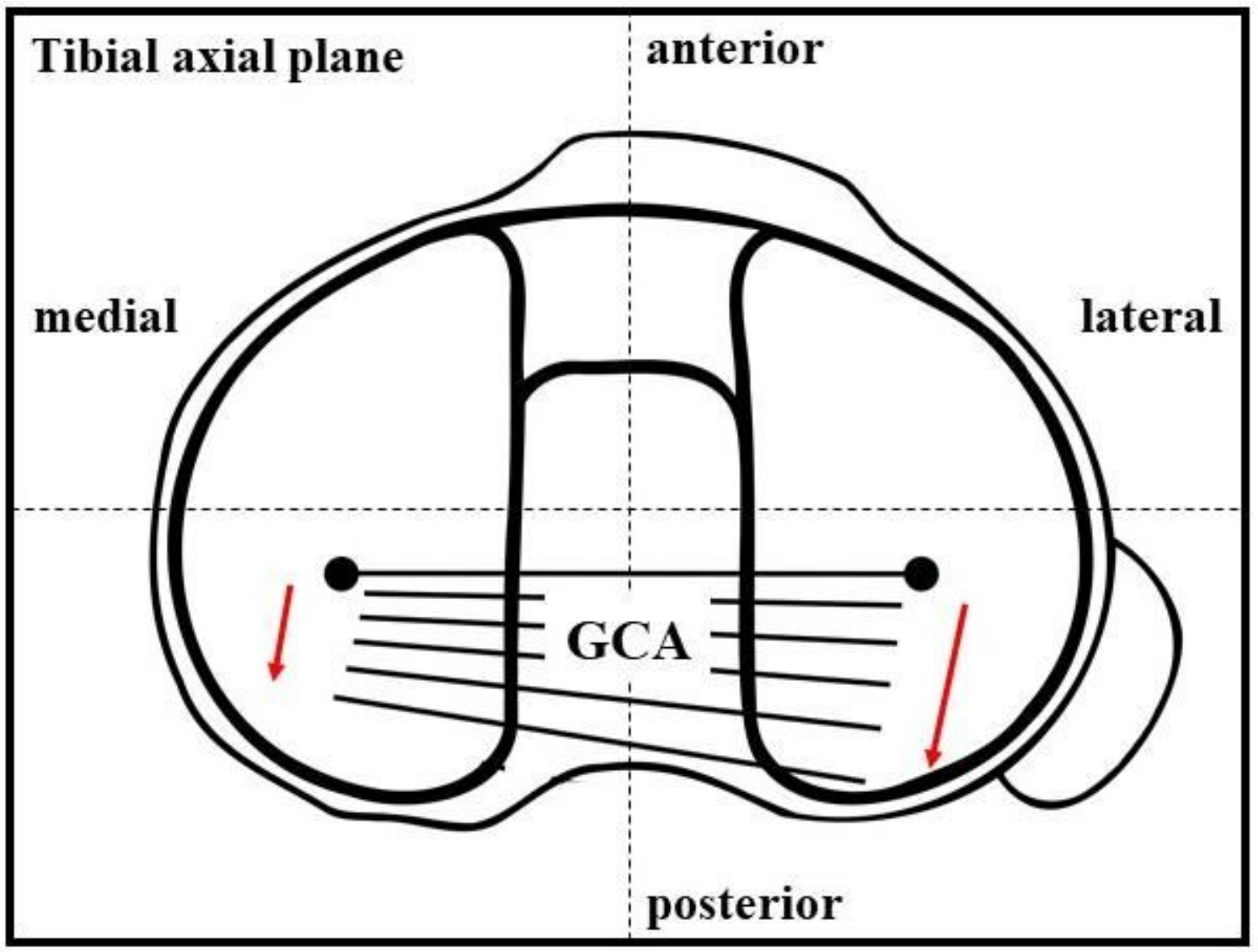


Figure 4

Parameters of kinematic evaluation of the knee. The femoral GCA was projected on the tibial axial plane and the medial and lateral endpoints of the GCA were evaluated based on the tibial coordinate value.

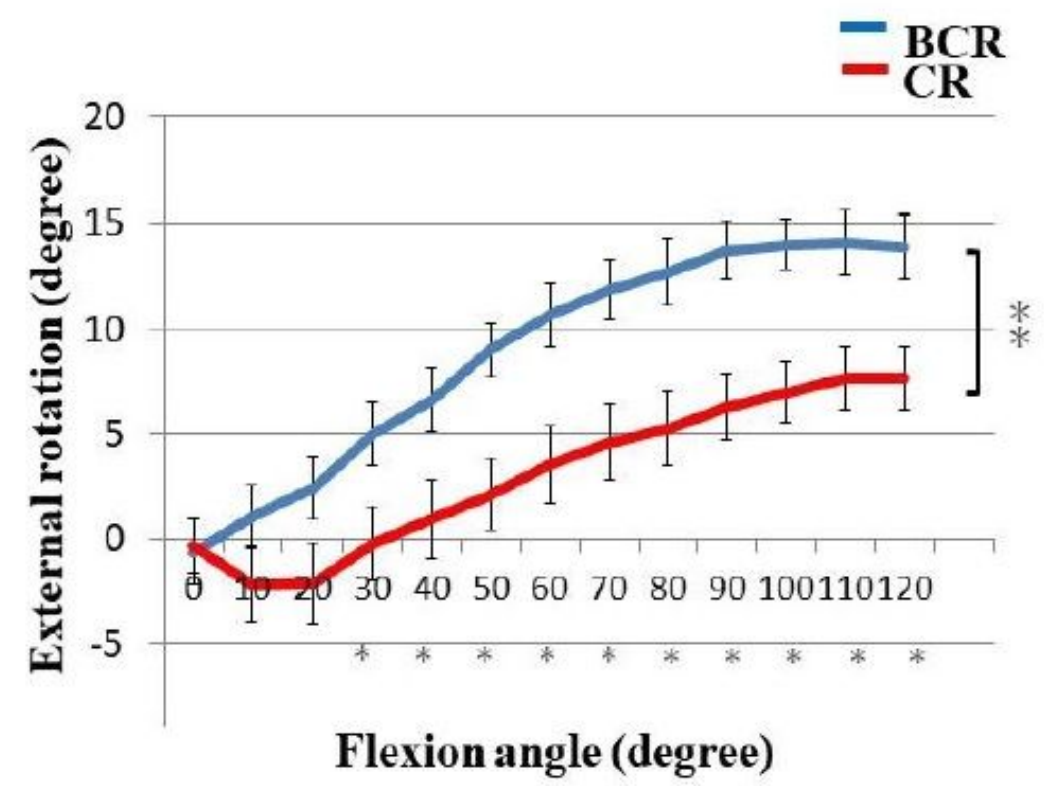

a

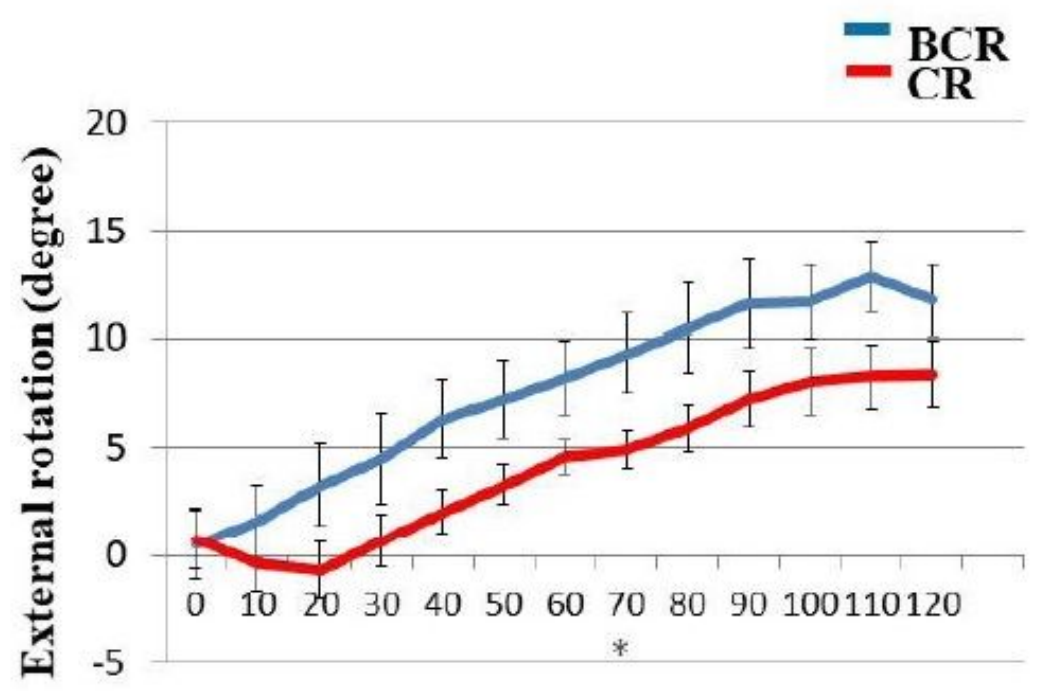

Flexion angle (degree)

$\mathrm{b}$

Figure 5 
Rotation angle of the femoral component with deep flexion. ${ }^{*} p<0.05$ by Mann-Whitney $U$ test for the difference between BCR-TKA and CR-TKA per flexion angle. ${ }^{*} \mathrm{p}<0.05$ by repeated measures ANOVA for the difference between BCR-TKA and CR-TKA across the flexion angles. a. On level ground. b. On a $10^{\circ}$ forward slope.

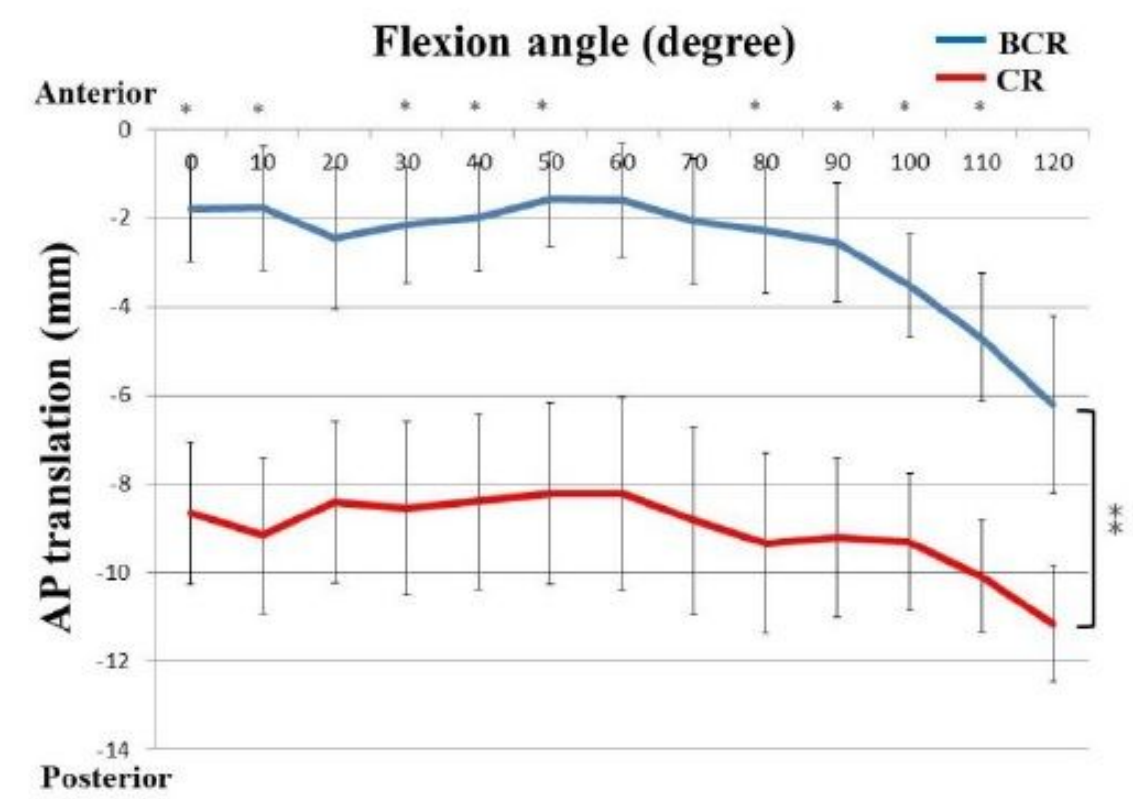

a.

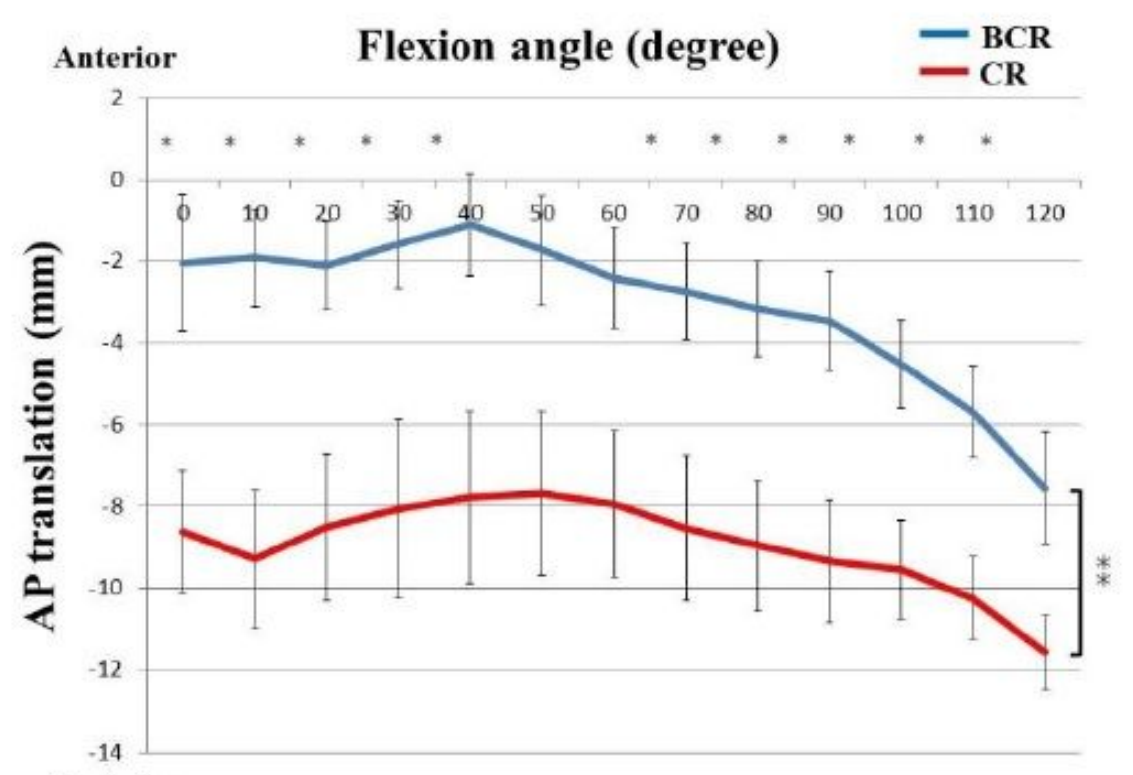

Posterior

b.

Figure 6 
Anteroposterior movement of the medial contact point of the femoral component with deep flexion. Anteroposterior (AP) translation of the femoral medial point during a single deep lunge. ${ }^{*} p<0.05$ by Mann-Whitney $U$ test for the difference between BCR-TKA and CR-TKA per flexion angle. ${ }^{\star \star} p<0.05$ by repeated measures ANOVA for the difference between BCR-TKA and CR-TKA across the flexion angles. a. On level ground. b. On a $10^{\circ}$ forward slope.

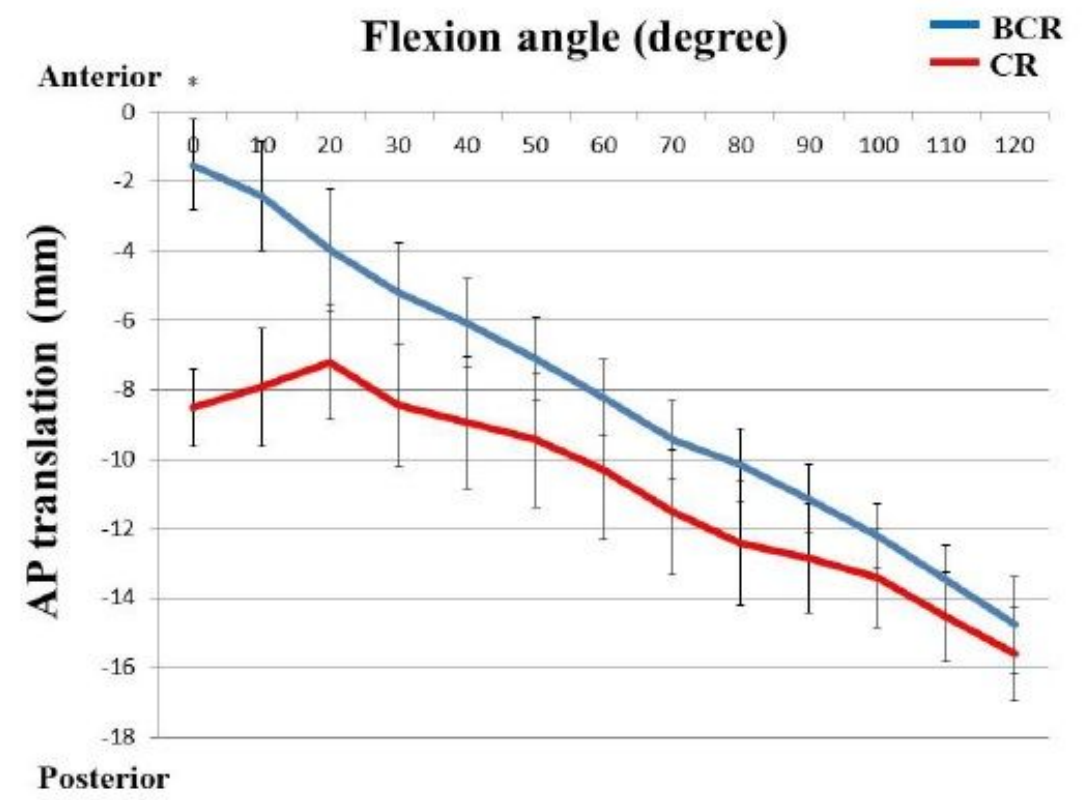

a.

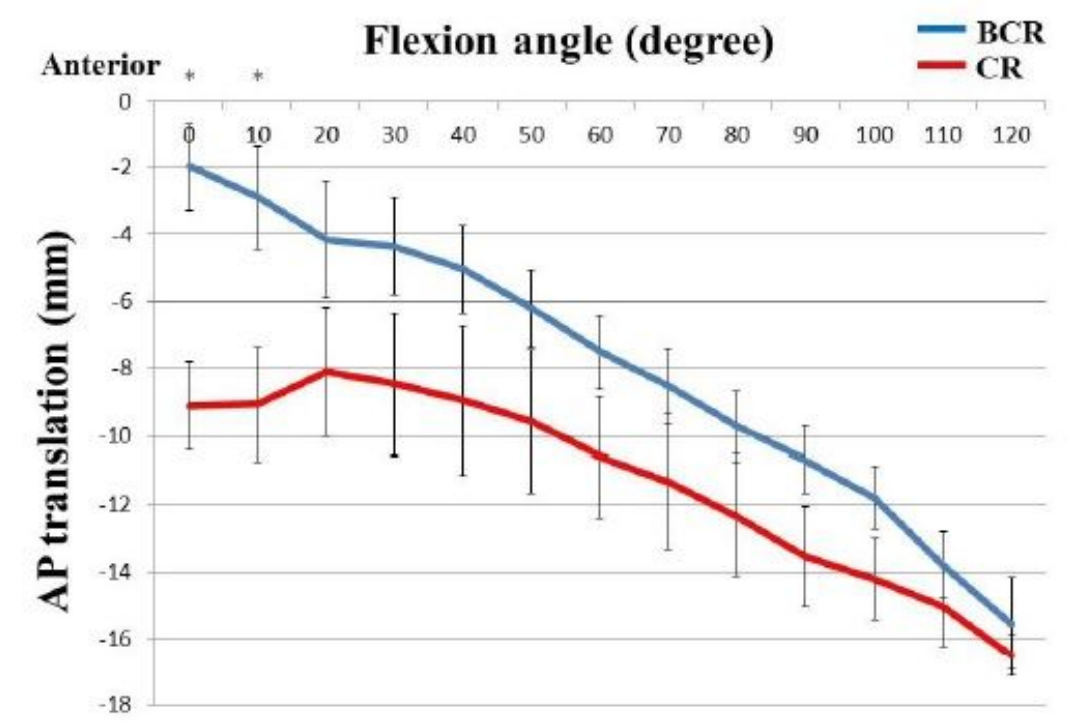

Posterior

b.

Figure 7 
Anteroposterior movement of the lateral contact point of the femoral component in deep flexion.

Anteroposterior (AP) translation of the femoral lateral epicondyle during a single deep lunge. ${ }^{*} p<0.05$ by Mann-Whitney $U$ test for the difference between BCR-TKA and CR-TKA per flexion angle. a. On level ground. b. On a $10^{\circ}$ forward slope.

\section{Supplementary Files}

This is a list of supplementary files associated with this preprint. Click to download.

- Table1.pdf 